\title{
A conserved extended signal peptide region directs posttranslational protein translocation via a novel mechanism
}

\author{
Mickaël Desvaux, ${ }^{1} \dagger$ Anthony Scott-Tucker, ${ }^{1}$ Sue M. Turner, ${ }^{1}$ \\ Lisa M. Cooper, ${ }^{1}$ Damon Huber, ${ }^{2}$ James P. Nataro ${ }^{3}$ and lan R. Henderson ${ }^{1}$ \\ ${ }^{1}$ Division of Immunity and Infection, University of Birmingham, Edgbaston, Birmingham B15 \\ 2TT, UK \\ ${ }^{2}$ Department of Microbiology and Molecular Genetics, Harvard Medical School, Boston, MA \\ 02115, USA \\ ${ }^{3}$ Center for Vaccine Development, University of Maryland, Baltimore, MD 21201, USA
}

Received 24 April 2006

Revised 24 September 2006

Accepted 25 September 2006

\begin{abstract}
Members of the type $V$ secretion family are among the most prevalent secreted proteins in Gramnegative bacteria. A subset of this family, including Pet, the prototypical member of the Enterobacteriaceae serine proteases, possess unusual signal peptides which can be divided into five regions termed N1 (charged), $\mathrm{H} 1$ (hydrophobic), N2, H2 and C (cleavage site) domains. The N1 and $\mathrm{H} 1$ regions, which the authors have named the extended signal peptide region (ESPR), demonstrate remarkable conservation. In contrast, the $\mathrm{N} 2, \mathrm{H} 2$ and $\mathrm{C}$ regions show significant variability, and are reminiscent of typical Sec-dependent signal sequences. Despite several investigations, the function of the ESPR remains obscure. Here, it is shown that proteins possessing the ESPR are translocated in a posttranslational fashion. The presence of the ESPR severely impairs inner membrane translocation. Mutational analysis suggests that the ESPR delays inner membrane translocation by adopting a particular conformation, or by interacting with a cytoplasmic or inner membrane co-factor, prior to inner membrane translocation.
\end{abstract}

\section{INTRODUCTION}

A major issue in the cell biology of all organisms is how proteins are targeted to the correct cellular compartment, particularly when it involves translocation across biological membranes. In Gram-negative bacteria, which possess two biological membranes, seven major pathways (type I-VI and the chaperone-usher pathways) are currently recognized as having evolved to permit the transport of soluble proteins to the extracellular milieu (Henderson et al., 2004; Mougous et al., 2006; Thanassi et al., 2005). One of these pathways is the type $V$ secretion system (T5SS), which can be subdivided into: (i) the T5aSS, which includes the classical autotransporters; (ii) the T5bSS, which includes the two-partner secretion pathway; and (iii) the T5cSS, representing the recently described trimeric autotransporters (Henderson et al., 2004).

tPresent address: Institut National de la Recherche Agronomique (INRA), Centre de Recherche Clermont-Ferrand - Theix - Lyon, UR 454 - Unité de Microbiologie, Site de Theix, F-63122 Saint-Genès Champanelle, France.

Abbreviations: EP-PCR, error-prone PCR; ESPR, extended signal peptide region; FHA, filamentous haemagglutinin; SPATE, serine protease autotransporter of the Enterobacteriaceae; SRP, signal recognition particle; T5SS, type $V$ secretion system; TU, translocation unit; XP, 5-bromo-4-chloro-3-indolyl phosphate.
The T5SS can be briefly defined as a system that permits translocation of proteins, which fold largely into $\beta$-helical structures, across the outer membrane via a $\beta$-barrel transmembrane pore, with outer membrane translocation occurring in the absence of an apparent energy input, i.e. ATP (Desvaux et al., 2004a, b; Henderson et al., 1998, 2004). Proteins secreted via the T5aSS and T5cSS are synthesized as a single modular polypeptide composed of three main domains: (i) an N-terminal signal peptide mediating inner membrane translocation; (ii) a central passenger domain corresponding to the functional portion of the molecule; and (iii) a C-terminal translocation unit (TU), which forms a $\beta$-barrel pore within the outer membrane, and permits translocation of the functional passenger domain to the bacterial cell surface and beyond (Henderson et al., 2004). These two systems are differentiated by the fact that members of the T5aSS possess a single intact TU, whereas members of the T5cSS possess only one third of a TU, and are required to trimerize to form a functional $\mathrm{TU}$ in the outer membrane (Cotter et al., 2005; Meng et al., 2006; Surana et al., 2004). In contrast, the exoproteins (designated TpsA) secreted via the T5bSS are synthesized separately from their $\beta$-barrel $\mathrm{TU}$ (designated TpsB). Both proteins possess signal sequences and are translocated independently across the inner membrane, and after translocation through 


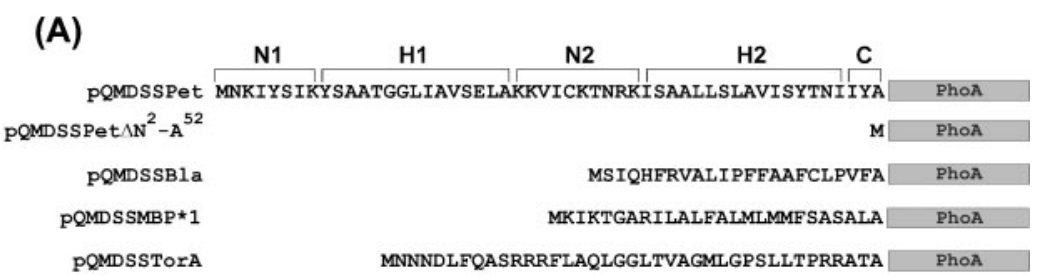

(B)

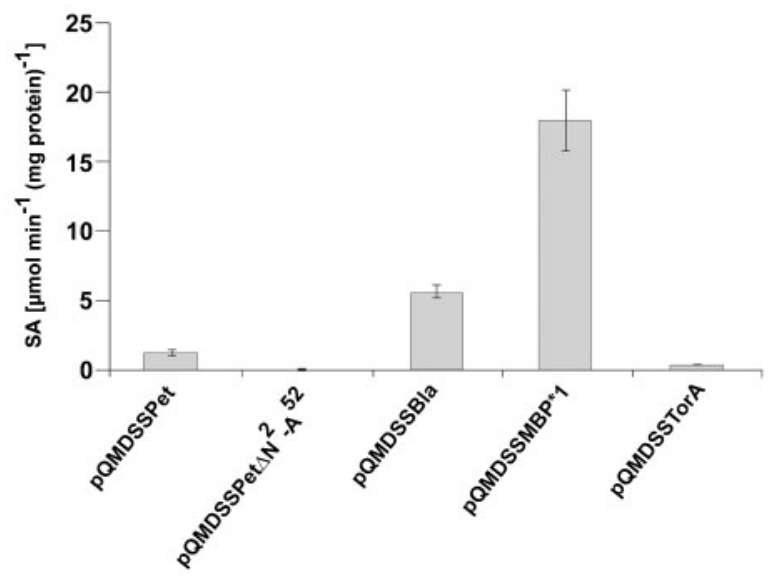

(C)

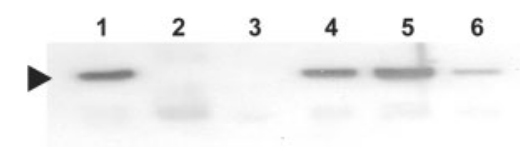

Fig. 1. Comparison of the translocation efficiency of the Pet signal peptide with that of other specific signal peptides. (A) Schematic depiction of the PhoA protein fusion constructs made in pQUANTagen $(k x)$ with various signal peptides. pQMDSSPet represents the Pet-extended signal sequence fused to PhoA. The locations of the N1, H1, N2, H2 and C domains are indicated. pQMDSSBla represents a fusion of the Bla signal peptide to PhoA. The Bla signal peptide permits protein targeting to $\mathrm{Sec}$ via an SRP-independent pathway (Beha et al., 2003). pQMDSSMBP*1 represents the $\mathrm{MBP}^{*} 1$ signal peptide fused to PhoA. The MBP*1 signal peptide allows Sec translocation in an SRP-dependent manner (Lee \& Bernstein, 2001). pQMDSSTorA represents the TorA signal peptide fused to PhoA. The TorA signal peptide is specific to the Tat pathway (Cristobal et al., 1999). (B) Specific activity (SA) of periplasmic PhoA expressed from the various signal peptidePhoA fusion constructs depicted in (A). Data are from three independent experiments, and error bars represent SD. The Bla and MBP fusions display higher levels of PhoA activity than the native Pet signal sequence fusion. (C) Western immunoblot analysis of total cellular protein derived from $E$. coli $\Delta$ Star harbouring the various signal peptide-PhoA fusion constructs, and probed with anti-PhoA antibodies. Lane 1, pQMDSSPet; lane 2, pQMDSSPet $\Delta N^{2}-A^{52}$; lane 3 , empty host strain; lane 4, pQMDSSBla; lane 5, pQMDSSMBP* 1 ; lane 6 , pQMDSSTorA. The arrow indicates the position of PhoA. Levels of PhoA were similar in the Pet, MBP and Bla signal sequence fusions, indicating that the higher levels of PhoA activity were due to the amount of translocated protein rather than the higher levels of expression. the inner membrane, undergo a specific interaction at the outer membrane before the exoprotein is translocated to the external milieu (Henderson et al., 2004; Jacob-Dubuisson et al., 2001, 2004; Newman \& Stathopoulos, 2004).

In the current model for T5SS, the signal peptide first targets the protein destined for secretion to the inner membrane Sec translocon (SecYEG), which then exports the protein into the periplasm (Henderson et al., 2004). The majority of proteins secreted via the T5SS have signal sequences that possess the canonical $\mathrm{N}$ (charged), $\mathrm{H}$ (hydrophobic) and C (signal peptidase cleavage site) domains associated with translocation via the classic posttranslational pathway (Henderson et al., 1998, 2004). However, several members of this family have been noted to have unusual signal peptides that exceed 50 aa in length (Desvaux et al., 2006; Henderson et al., 1998). While these unusual signal peptides do possess the canonical domains associated with signal sequences of the posttranslational pathway (N2, H2 and C), they are distinguished by an $\mathrm{N}$-terminal extension, which possesses an additional charged domain (N1) and an additional hydrophobic domain (H1) designated the extended signal peptide region (ESPR) (Fig. 1). Interestingly, and in contrast to the normal posttranslational signal peptides, which are highly degenerate, the N-terminal extensions of these signal peptides possess a high degree of conservation (Desvaux et al., 2006; Henderson et al., 1998, 2004). Such conservation is often indicative of a conserved function, and suggests that these N-terminal extensions play an important role in the biogenesis of proteins secreted via the T5SS.

Investigations have suggested that the unusual signal peptides of Hbp and EspP, both members of the serine protease autotransporters of the Enterobacteriaceae (SPATE) 
subfamily, target proteins to the signal recognition particle (SRP) pathway (Peterson et al., 2003; Sijbrandi et al., 2003). Further investigations have indicated that deletion of the ESPR inhibits passenger domain translocation across the outer membrane, and that overproduction of proteins harbouring the ESPR affects the translocation efficiency of Sec-dependent proteins (Szabady et al., 2005). In contrast, in vitro and in vivo investigations of filamentous haemagglutinin (FHA), a TpsA protein harbouring an ESPR, have demonstrated that SecA and SecB are required for inner membrane translocation via the posttranslational pathway, and that, despite cross-linking with SRPs, inner membrane translocation is strictly SRP-independent (Chevalier et al., 2004). Moreover, it has been noted that rather than determining a specific mode of membrane targeting and translocation, the extended signal peptide of FHA influences the rate of inner membrane translocation (Chevalier et al., 2004). Thus, the exact nature of the inner membrane translocation event remains controversial.

To investigate the structure-function relationship of the unusual signal peptide, and in particular the ESPR region, we studied Pet, a prototypical member of the SPATE subfamily (Eslava et al., 1998; Henderson \& Nataro, 2001; Henderson et al., 2004). In the current study, we provide evidence that an ESPR-containing signal sequence directs protein translocation across the inner membrane in a novel posttranslational fashion, and that the presence of the ESPR strongly affects the efficiency of inner membrane translocation.

\section{METHODS}

Bacterial strains, plasmids and culture conditions. The bacterial strains and plasmids used in this study are listed in Table 1. Strains were passaged routinely on LB broth or agar with the following supplements where appropriate: ampicillin $\left(100 \mu \mathrm{g} \mathrm{ml}^{-1}\right)$, kanamycin $\left(50 \mu \mathrm{g} \mathrm{ml}^{-1}\right)$, nalidixic acid $\left(50 \mu \mathrm{g} \mathrm{ml}^{-1}\right)$, 5-bromo-4chloro-3-indolyl phosphate (XP; $40 \mu \mathrm{g} \mathrm{ml}^{-1}$ ) and IPTG $(0.5 \mathrm{mM})$. The plasmid pCEFN1 overexpressing Pet has been described previously (Eslava et al., 1998). pQUANTagen(kx) (Quantum Appligene) was used to construct PhoA reporter fusions.

Protein purification and analysis. Culture supernatant fractions were prepared as described previously (Eslava et al., 1998). Protein concentrations were calculated using the Bradford dye method (Bradford, 1976). 1D SDS-PAGE was performed by using $12.5 \%$ (w/v) acrylamide resolving gels and $4.5 \%(\mathrm{w} / \mathrm{v})$ acrylamide stacking gels (Laemmli, 1970). Proteins were detected by staining with Coomassie brilliant blue R250 or the SilverQuest Silver Staining kit (Invitrogen), as described by the supplier. For Western immunoblotting, horseradish peroxidase-conjugated goat anti-rabbit IgG (Sigma) was used as the localizing reagent, and reacting bands were visualized using the SuperSignal West Pico Chemiluminescent Substrate according to the manufacturer's instructions (Pierce).

DNA manipulations and sequencing. Standard molecular biology procedures and DNA manipulations were used (Sambrook \& Russell, 2001). Plasmids were purified using the QIAprep Spin Miniprep kit (Qiagen). DNA purification from PCR/enzymic reactions and agarose gels were performed using the QIAquick PCR Purification and Gel Extraction kits (Qiagen), respectively.
Restriction enzymes and T4 DNA ligase from Invitrogen were used. PCR experiments were performed using Platinum Pfx DNA Polymerase (Invitrogen). Site-directed mutations were constructed with the QuikChange II kit (Stratagene). Error-prone PCR (EPPCR) was performed using the GeneMorph II Random Mutagenesis kit (Stratagene), to achieve 1-3 mutations per kilobase. All of the above manipulations were carried out in accordance with the instructions of the respective manufacturers. PCR and sequencing primers are listed in Table 2.

Site-directed mutagenesis of the Pet signal peptide. The highly conserved residues within the ESPR were mutated to nonconservative residues, using site-directed mutagenesis with pCEFN1 as the template. Using primers IRHM1F and IRHM1R, a plasmid $\left(\mathrm{pCEFN} 1-\mathrm{M}^{1} \rightarrow^{*}\right.$ ) was constructed in which the putative initiation codon was converted to a stop codon (TAG). Similarly, pCEFN1$\mathrm{N}^{2} \rightarrow \mathrm{S}^{2}$ (primers IRHN2F and IRHN2R), pCEFN1- $\mathrm{Y}^{5} \rightarrow \mathrm{R}^{5}$ (primers IRHY5F and IRHY5R), pCEFN1- $\mathrm{Y}^{9} \rightarrow \mathrm{R}^{9}$ (primers IRHY9F and IRHY9R) and pCEFN1-S ${ }^{20} \mathrm{EL} \rightarrow \mathrm{R}^{20} \mathrm{DR}$ (primers IRHS20F and IRHS20R) were constructed using the designated primers. Plasmids were transformed into Escherichia coli HB101.

Pulse-chase labelling. Pulse-chase labelling was performed as described by Ulbrandt et al. (1997). Briefly, E. coli DO251 and its isogenic secA mutant BA13 were transformed with pQMDSSPet, and grown overnight at $30^{\circ} \mathrm{C}$ in $\mathrm{M} 9$ medium containing ampicillin, $0.4 \%(\mathrm{w} / \mathrm{v})$ glucose, and $40 \mu \mathrm{g}$ L-amino acids $\mathrm{ml}^{-1}$, excluding methionine and cysteine. Cells were resuspended in fresh medium at $\mathrm{OD}_{550}$ 0.02, and grown for $3 \mathrm{~h}$. Overexpression of PhoA fusion proteins was induced by the addition of IPTG 30 min prior to labelling. Aliquots were removed and cells were pulse-labelled for $30 \mathrm{~s}$ with $30 \mu \mathrm{Ci} \mathrm{ml}{ }^{-1}$ (1.1 MBq ml ${ }^{-1}$ ) $\operatorname{Tran}^{35}$ S-label (ICN Biochemicals). Cold methionine and cysteine $(1 \mathrm{mM})$ were then added for the chase. Cold TCA was added at various times to a final concentration of $10 \%$. Precipitates were collected by centrifugation $(16000 \mathrm{~g}$, for $10 \mathrm{~min}$ ), and pellets were redissolved in solubilization buffer. Samples were diluted 1:20 into radioimmunoprecipitation (RIPA) buffer and aliquots were withdrawn for immunoprecipitation by anti-PhoA antibodies. After washing, immunoprecipitated proteins were resolved by SDS-PAGE and visualized using a Fuji BAS2000 phosphorimager.

\section{Construction and analysis of thioredoxin reporter constructs.} The pet signal sequence, incorporating three residues of mature Pet in order to maintain the signal peptidase cleavage site, was amplified using primers AST1 and AST2, incorporating $B s p H I$ and $N c o I$ restriction sites at the $5^{\prime}$ and $3^{\prime}$ ends, respectively. To create pPetSSTrxA, the digested PCR product was ligated to a digested inverse PCR product of the backbone of plasmid pCFS123, generated using primers AST3 and AST4, such that the plasmid expressed an in-frame fusion between the Pet signal sequence and thioredoxin (PetSS-TrxA). TrxA expression was monitored as previously described (Huber et al., 2005).

Construction and analysis of PhoA fusions. To monitor the ability of the Pet signal sequence and mutant derivatives to mediate inner membrane translocation, a reporter system, under the control of an IPTG-inducible ptac promoter, was constructed using pQUANTagen $(\mathrm{kx})$. Chloramphenicol amplification was used for pQUANTagen $(\mathrm{kx})$ isolation (Sambrook \& Russell, 2001). Fractions were harvested from mid-exponential-phase cultures after treatment with $100 \mathrm{mM}$ iodoacetamide (DeLisa et al., 2003) and combined lysozyme/osmotic shock treatment (French et al., 1996). Alkaline phosphatase activity of PhoA was assayed spectrophotometrically, utilizing $p$-nitrophenyl phosphate as a substrate at $25^{\circ} \mathrm{C}$, by monitoring the release of $p$-nitrophenolate at $410 \mathrm{~nm}$ (Walter \& Schutt, 1976). Specific activity was determined in a range in which a linear relationship with protein concentration was established. Results were 
Table 1. Strains and plasmids used in this study

\begin{tabular}{|c|c|c|}
\hline Strain or plasmid & Relevant description & Reference \\
\hline \multicolumn{3}{|l|}{ Strains } \\
\hline E. coli TOP10 & 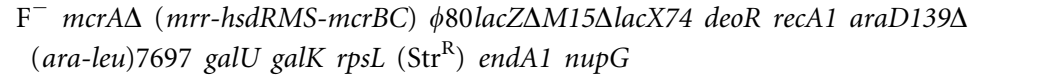 & Invitrogen \\
\hline E. coli $\mathrm{HB} 101$ & $\begin{array}{l}\mathrm{F}^{-} \text {thi-1 hsdS20 }\left(r B^{-}, m B^{-}\right) \text {supE44 recA13 ara-14 leuB6 proA2 lacY1 galK2 } \\
\text { rpsL20 }\left(\mathrm{Str}^{\mathrm{R}}\right) \text { xyl-5 mtl-1 }\end{array}$ & Promega \\
\hline E. coli $\Delta$ Star & $\Delta p h o A$ & Quantum Appligene \\
\hline E. coli WP570 & $\Delta \operatorname{tr} x A$ & Schierle et al. (2003) \\
\hline E. coli $\mathrm{B} 1 \mathrm{LKO}$ & $\Delta$ tatC & Bogsch et al. (1998) \\
\hline \multicolumn{3}{|l|}{ Plasmids } \\
\hline pCEFN1 & pSPORT1 derivative expressing Pet protein from its original promoter; $A_{m p}{ }^{R}$ & Eslava et al. (1998) \\
\hline pPetSStrxA & PetSS-TrxA fusion & This study \\
\hline pCFS123 & DsbASS-TrxA fusion & Schierle et al. (2003) \\
\hline pCFS126 & PhoASS-TrxA fusion & Schierle et al. (2003) \\
\hline pCEFN1-M $\rightarrow^{*}$ & Site-directed mutation in pCEFN1 where $\mathrm{M}^{1}$ of Pet is changed to a stop codon & This study \\
\hline pCEFN1-N ${ }^{2} \rightarrow S^{2}$ & Site-directed mutation in pCEFN1 where $\mathrm{N}^{2}$ of Pet is changed to $\mathrm{S}^{2}$ & This study \\
\hline $\mathrm{pCEFN} 1-\mathrm{Y}^{5} \rightarrow \mathrm{R}^{5}$ & Site-directed mutation in pCEFN1 where $\mathrm{Y}^{5}$ of Pet is changed to $\mathrm{R}^{5}$ & This study \\
\hline pCEFN $1-\mathrm{Y}^{9} \rightarrow \mathrm{R}^{9}$ & Site-directed mutation in pCEFN1 where $\mathrm{Y}^{9}$ of Pet is changed to $\mathrm{R}^{9}$ & This study \\
\hline pCEFN1-S ${ }^{20} E L \rightarrow R^{20} D R$ & Site-directed mutation in pCEFN1 where $\mathrm{S}^{20} \mathrm{EL}$ of Pet is changed to $\mathrm{R}^{20} \mathrm{DR}$ & This study \\
\hline pQUANTagen $(\mathrm{kx})$ & phoA, p15A origin of replication, promoter ptac; $\mathrm{Amp}^{\mathrm{R}}$ & Quantum Appligene \\
\hline pQMDSSPet & Pet signal sequence cloned into pQUANTagen $(\mathrm{kx})$ & This study \\
\hline pQMDSSPet $\Delta N^{2}-A^{52}$ & PhoA without any signal sequence, but containing the methionine initiation codon & This study \\
\hline pQMDSSBla & $\beta$-Lactamase signal sequence cloned into pQUANTagen $(\mathrm{kx})$ & This study \\
\hline pQMDSSMBP* 1 & $\begin{array}{l}\text { Triple-point-mutated maltose-binding protein (MBP) signal peptide cloned into } \\
\text { pQUANTagen }(\mathrm{kx})\end{array}$ & This study \\
\hline pQMDSSTorA & TorA signal sequence cloned into pQUANTagen $(\mathrm{kx})$ & This study \\
\hline pQMDSSPetM ${ }^{1} \rightarrow^{*}$ & pQMDSSPet with $\mathrm{M}^{1}$ from Pet signal sequence mutated into a stop codon & This study \\
\hline pQMDSSPetM ${ }^{1} \rightarrow I^{1}$ & pQMDSSPet with $\mathrm{M}^{1}$ from Pet signal sequence mutated into $\mathrm{I}^{1}$ & This study \\
\hline pQMDSSPetM ${ }^{1} \rightarrow \mathrm{L}^{1}$ & pQMDSSPet with $\mathrm{M}^{1}$ from Pet signal sequence mutated into $\mathrm{L}^{1}$ & This study \\
\hline pQMDSSPetM ${ }^{1} \rightarrow K^{1}$ & pQMDSSPet with $\mathrm{M}^{1}$ from Pet signal sequence mutated into $\mathrm{K}^{1}$ & This study \\
\hline pQMDSSPetN ${ }^{2} \rightarrow \mathrm{A}^{2}$ & pQMDSSPet with $\mathrm{N}^{2}$ from Pet signal sequence mutated into $\mathrm{A}^{2}$ & This study \\
\hline pQMDSSPet $Y^{9} \rightarrow \mathrm{D}^{9}$ & pQMDSSPet with $\mathrm{Y}^{9}$ from Pet signal sequence mutated into $\mathrm{D}^{9}$ & This study \\
\hline pQMDSSPetE ${ }^{21} \rightarrow \mathrm{A}^{21}$ & pQMDSSPet with $E^{21}$ from Pet signal sequence mutated into $A^{21}$ & This study \\
\hline pQMDSSPetL ${ }^{41} \rightarrow \mathrm{P}^{41}$ & pQMDSSPet with $\mathrm{L}^{41}$ from Pet signal sequence mutated into $\mathrm{P}^{41}$ & This study \\
\hline pQMDSSPetL $^{41} \rightarrow \mathrm{Q}^{41}$ & pQMDSSPet with $\mathrm{L}^{41}$ from Pet signal sequence mutated into $\mathrm{Q}^{41}$ & This study \\
\hline pQMDSSPet $\Delta \mathrm{N}^{2}-\mathrm{I}^{7}$ & pQMDSSPet with deletion of $\mathrm{N}^{2}-\mathrm{I}^{7}$ region in Pet signal sequence & This study \\
\hline pQMDSSPet $\Delta \mathrm{N}^{2}-\mathrm{I}^{17}$ & pQMDSSPet with deletion of $\mathrm{N}^{2}-\mathrm{I}^{17}$ region in Pet signal sequence & This study \\
\hline pQMDSSPet $\Delta N^{2}-A^{23}$ & pQMDSSPet with deletion of $\mathrm{N}^{2}-\mathrm{A}^{23}$ region in Pet signal sequence & This study \\
\hline pQMDSSPet $\Delta \mathrm{N}^{2}-\mathrm{I}^{27}$ & pQMDSSPet with deletion of $\mathrm{N}^{2}-\mathrm{I}^{27}$ region in Pet signal sequence & This study \\
\hline pQMDSSPet $\Delta \mathrm{A}^{11}-\mathrm{A}^{23}$ & pQMDSSPet with deletion of $A^{11}-A^{23}$ region in Pet signal sequence & This study \\
\hline pQMDSSPet $\Delta \mathrm{K}^{24}-\mathrm{K}^{33}$ & pQMDSSPet with deletion of $\mathrm{K}^{24}-\mathrm{K}^{33}$ region in Pet signal sequence & This study \\
\hline pQMDSSPet $\Delta \mathrm{I}^{34}-\mathrm{I}^{49}$ & pQMDSSPet with deletion of $\mathrm{I}^{34}-\mathrm{I}^{49}$ region in Pet signal sequence & This study \\
\hline
\end{tabular}

expressed in $\mu \mathrm{mol} \mathrm{min}^{-1}$ (mg protein $)^{-1}$, using a molar extinction coefficient of $18300 \mathrm{M} \mathrm{cm}^{-1}$ for $p$-nitrophenolate. Data were derived from three independent experiments, and were analysed by Student's $t$ test for unpaired data, to determine statistically significant differences $(P<0.05)$.

The Pet signal peptide was amplified from pCEFN1 using primers SSPetBamFw and SSPetSacRv, and then cloned into pQUANTagen $(\mathrm{kx})$, resulting in plasmid pQMDSSPet. Sequential deletions from the Nterminal end of the Pet signal peptide were achieved by PCR from pQMDSSPet, using the reverse primer SSPetSacRv and the forward primers SSPetDeletN2-I7BamFw, SSPetDeletN2-I17BamFw, SSPetDeletN2-A23BamFw and SSPetDeletN2-I27BamFw. The respective plasmid constructs pQMDSSPet $\Delta \mathrm{N}^{2}-\mathrm{I}^{7}, \quad$ pQMDSSPet $\Delta \mathrm{N}^{2}-\mathrm{I}^{17}$, pQMDSSPet $\Delta \mathrm{N}^{2}-\mathrm{A}^{23}$ and pQMDSSPet $\Delta \mathrm{N}^{2}-\mathrm{I}^{27}$ were obtained after cloning of the products into pQUANTagen $(\mathrm{kx})$. pQMDSSPet $\Delta \mathrm{I}^{34}-\mathrm{I}^{49}$ was obtained after cloning PCR products from pQMDSSPet, using the forward primer SSPetBamFw and the reverse primer SSPetDeletI34I49SacRv, into pQUANTagen $(\mathrm{kx})$.

Internal deletions were constructed by inverse PCR using pQMDSSPet as a template. Primers IPCRSSPetDeletA11-A23NdeFw and IPCRSSPetDeletA11-A23NdeRv were used to create a signal sequence-PhoA construct ( $\mathrm{pQMDSSPet} \Delta \mathrm{A}^{11}-\mathrm{A}^{23}$ ) lacking the $\mathrm{H} 1$ domain, and primers IPCRSSPetDeletK24-K33SfiFw and IPCRSSPetDeletK24-K33SfiRv were used to create a signal 
Table 2. Primers used in this study

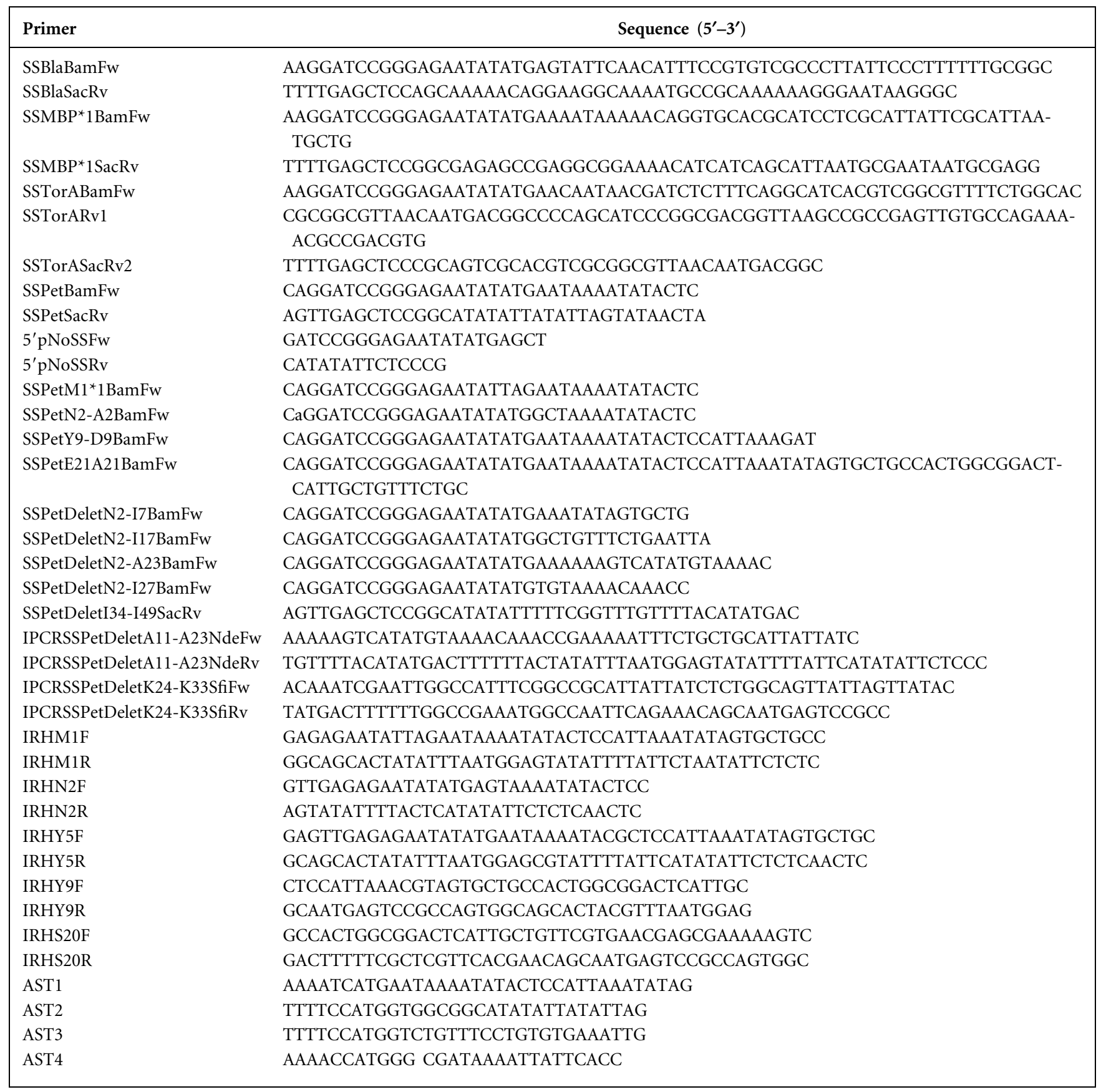

sequence-PhoA construct ( $\mathrm{pQMDSSPet} \Delta \mathrm{K}^{24}-\mathrm{K}^{33}$ ) lacking the $\mathrm{N} 2$ domain. Linear DNA products from the inverse PCR reactions were digested with the appropriate restriction enzyme (either NdeI or SfiI) before ligation.

To construct pQMDSSPet $\Delta \mathrm{M}_{1}-\mathrm{A}_{52}$, dsDNA was obtained by mixing equimolar amounts of $5^{\prime}$-phosphorylated primers $5^{\prime}$ pNoSSFw and $5^{\prime} \mathrm{pNoSSRv}$ dissolved in annealing buffer $(10 \mathrm{mM}$ Tris, $\mathrm{pH} 8.0$, $50 \mathrm{mM} \mathrm{NaCl}, 1 \mathrm{mM}$ EDTA), and then heating at $95^{\circ} \mathrm{C}$ and gradually cooling to room temperature before cloning into pQUANTagen $(\mathrm{kx})$ pre-digested with $\mathrm{BamHI} / \mathrm{SacI}$. The $\beta$-lactamase (SSBlaBamFw and SSBlaSacRv) and $\mathrm{MBP}^{\star} 1$ (SSMBP${ }^{\star} 1 \mathrm{BamFw}$ and $\left.\mathrm{SSMBP}^{\star} 1 \mathrm{SacRv}\right)$ signal peptides were synthesized without a DNA template, following
PCR using the indicated primer pairs, which resulted in pQMDSSBla and $\mathrm{pQMDSSMBP}{ }^{\star} 1$ after cloning in pQUANTagen $(\mathrm{kx})$. The TorA signal peptide was similarly synthesized using primers SSTorABamFw and SSTorARv1, but with a nested PCR using primers SSTorABamFw and SSTorASacRv2, before cloning to create pQMDSSTorA.

Random and site-directed mutagenesis of the Pet signal sequence was performed using $\mathrm{PQMDSSPet}$ as a template. For random mutagenesis, a 1112 bp fragment was amplified by EP-PCR using primers EPPCRFw and EPPCRRv, and the products were cloned into pQUANTagen $(\mathrm{kx})$. Mutagenesis of the positions (i) $\mathrm{M}^{1}$ into a stop codon (ATG $\rightarrow$ TAG), (ii) $\mathrm{N}^{2}$ into $\mathrm{A}^{2}(\mathrm{AAT} \rightarrow \mathrm{GCT})$, (iii) $\mathrm{Y}^{9}$ into $\mathrm{D}^{9}(\mathrm{TAT} \rightarrow \mathrm{GAT})$, and (iv) $\mathrm{E}^{21}$ into $\mathrm{A}^{21}(\mathrm{GAA} \rightarrow \mathrm{GCA})$ was performed by PCR with the reverse 
primer SSPetSacRv and the forward primers SSPetM1*1BamFw, SSPetN2A2BamFw, SSPetY9D9BamFw and SSPetE21A21BamFw, respectively (Table 2 ). The resulting PCR products were cloned into pQUANTagen $(\mathrm{kx})$, resulting in pQMDSSPetM $^{1} \rightarrow{ }^{*}, \mathrm{pQMDSSPet}^{2} \rightarrow$ $\mathrm{A}^{2}$, pQMDSSPet $\mathrm{Y}^{9} \rightarrow \mathrm{D}^{9}$ and $\mathrm{pQMDSSPet} \mathrm{E}^{21} \rightarrow \mathrm{A}^{21}$, respectively.

\section{RESULTS}

\section{Signal sequences possessing the ESPR direct inner membrane translocation}

The initial description of Pet suggests that this protein possesses an unusually long signal peptide of 52 aa spanning residues $\mathrm{M}^{1}-\mathrm{A}^{52}$ (Eslava et al., 1998). To assess the ability of this putative extended signal sequence to translocate proteins across the inner membrane, a heterologous reporter construct (pQMDSSPet) was created in which the signal sequence of PhoA, whose expression was governed by an IPTG-inducible ptac promoter, was replaced with the 52 aa of the putative Pet signal sequence (Fig. 1). Since PhoA is active only after it has been translocated to the periplasm, it provides a method of determining the efficiency of inner membrane translocation. After induction with IPTG, PhoA activity could be detected from E. coli $\triangle$ Star pQMDSSPet, indicating that the ESPR-containing signal sequence acted as a signal peptide (Fig. 1). In contrast, E. coli $\Delta$ Star carrying a construct pQMDSSPet $\Delta \mathrm{N}^{2}-\mathrm{A}^{52}$ lacking residues $\mathrm{N}^{2}-\mathrm{A}^{52}$ of the Pet signal sequence demonstrated no PhoA activity or detectable levels of PhoA, as assessed by specific-activity measurements and Western immunoblotting, presumably due to the rapid degradation of the mistargeted protein (Fig. 1).

To determine the efficiency of the Pet signal sequence in directing inner membrane translocation of proteins, PhoA reporter constructs were created with signal peptides representative of different targeting pathways. Thus, the signal peptides from $\beta$-lactamase (as a model for protein routing towards a Sec-dependent, but SRP-independent pathway), $\mathrm{MBP}^{\star} 1$ (a triple-point mutant of the MBP signal peptide, used as a model of SRP-dependent signal peptides), and TorA (a model of a strictly Tat-dependent pathway) were fused to PhoA, and the specific activity of PhoA was calculated for each construct (Beha et al., 2003; Cristobal et al., 1999; DeLisa et al., 2003; Lee \& Bernstein, 2001) (Fig. 1). After the addition of IPTG, the Pet signal sequence fusion (PetSS-PhoA) demonstrated PhoA activity approximately 2.5 -fold higher than that of the TorA signal sequence fusion (TorASS-PhoA). However, Western immunoblotting revealed that the presence of detectable PhoA was severely diminished in strains possessing the TorASS-PhoA fusion (Fig. 1). This phenomenon has previously been observed, and the presence of active PhoA is attributed to leakage of Tat signal sequence fusions of Sec substrates through the Sec pathway, and to folding quality control of the Tat pathway (Richter \& Bruser, 2005). In contrast, the $\mathrm{MBP}^{\star} 1$ and $\beta$-lactamase signal sequences resulted in higher levels of PhoA activity, even though similar levels of PhoA were present, indicating that the differences in PhoA activity were due to differences in the amount of active translocated enzyme (Fig. 1).

\section{ESPR-containing signal sequences direct inner membrane translocation posttranslationally}

As we determined that the unusual signal peptide predicted for Pet was capable of directing inner membrane translocation, we wished to determine whether Pet was translocated via the SecYEG complex. The E. coli strains DO251 $\left(\mathrm{sec}^{+}\right.$ $\left.\sup F^{\text {ts }}\right)$ and BA13 $\left(\sec A^{\text {am }} \operatorname{supF^{\text {ts}}}\right)$ were transformed with pQMDSSPet and grown in minimal medium. Cells were subjected to pulse-chase labelling after the addition of IPTG and a shift to $41^{\circ} \mathrm{C}$, which switches off expression of SecA in strain BA13. Consistent with the hypothesis that the signal sequence directs inner membrane translocation via the SecYEG translocon, depletion of SecA had a pronounced effect on the accumulation of mature PhoA by inhibiting the cleavage of the Pet signal sequence from PhoA (Fig. 2A). In contrast, in the control strain DO251, the signal sequence

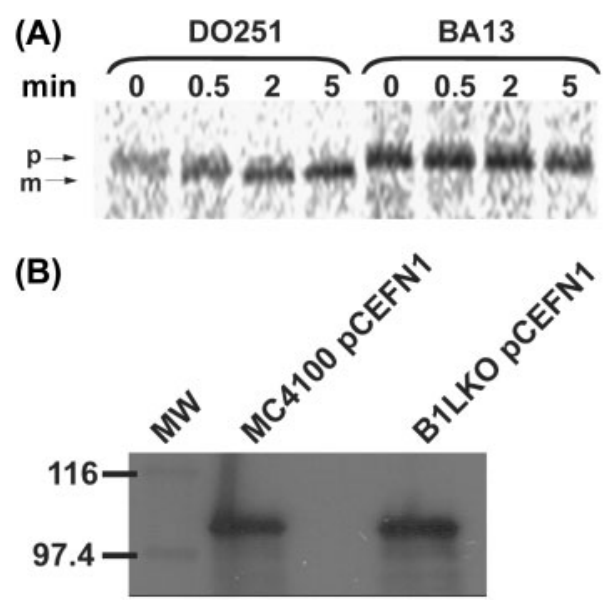

Fig. 2. Translocation pathways for an ESPR-containing protein across the inner membrane. (A) SecA depletion completely blocked translocation of proteins by an ESPR-containing signal peptide. E. coli BA13 and DO251 transformed with pQMDSSPet were grown as described in Methods. Processing of the Pet signal sequence was monitored after a temperature shift to $41^{\circ} \mathrm{C}$ to inactivate SecA expression in strain BA13. The processing of the Pet signal sequence and subsequent accumulation of mature PhoA were examined by pulse-chase analysis. The time length (min) of the chase is indicated. The Pet signal sequence was removed in the wild-type strain $E$. coli DO251, but no processing was evident in the SecA mutant strain $E$. coli BA13, indicating that the protein utilizes the energy input from SecA to pass through the SecYEG translocon. The position of the precursor $(p)$ and mature (m) forms of PhoA are indicated. (B) The tat $\mathrm{C}$ mutant and isogenic parent were transformed with pCEFN1, and the levels of secreted Pet in culture supernatants were monitored by SDS-PAGE. Pet was secreted efficiently in both strains, indicating that the Tat pathway does not play a major role in secretion of Pet. The sizes of the molecular mass markers (MW) are indicated in $\mathrm{kDa}$. 
was rapidly cleaved and mature PhoA was detected (Fig. 2A).

Most proteins possessing signal sequences which are targeted for secretion are translocated across the inner membrane in a posttranslational fashion; however, it has previously been suggested that the unusual signal sequences can target proteins for inner membrane translocation via an alternative pathway, such as the cotranslational SRP or the Tat pathways (Henderson et al., 1998). To determine if the Tat pathway played a role in inner membrane translocation, E. coli MC4100 and its isogenic tatC mutant E. coli B1LKO were transformed with either pQMDSSPet or pCEFN1 expressing native Pet. A significant role for the Tat pathway could be ruled out, as the levels of Pet secretion and PhoA activity were unaltered in the $t a t C$ mutant compared with those in the parent strain (Fig. 2B).

In contrast, SRP has been proposed to mediate targeting and inner membrane translocation of ESPR-containing homologues of Pet, namely, the SPATE autotransporters Hbp and EspP (Peterson et al., 2003; Sijbrandi et al., 2003). However, controversy has arisen during investigation of FHA, a TpsA protein harbouring an ESPR-containing signal peptide, demonstrating that despite cross-linking transiently with SRP, inner membrane translocation is trigger factor- and SecB-dependent, and strictly SRP-independent (Chevalier et al., 2004). Thus, it remains to be unequivocally determined whether ESPR-containing signal peptides direct posttranslational or cotranslational translocation across the inner membrane. Due to problems associated with depletion experiments, which have given rise to the above controversy, we sought to investigate the role of the ESPR in inner membrane targeting and translocation by using a reporter fusion based on the cytoplasmic protein thioredoxin. It has previously been demonstrated that thioredoxin is a rapidly folding protein which can only be efficiently translocated to the periplasm in the presence of a cotranslational signal peptide; posttranslational translocation is prevented by the rapid folding of thioredoxin in the cytoplasm, and the inability of the SecYEG translocon to accommodate folded proteins (Huber et al., 2005; Schierle et al., 2003). The DNA encoding the Pet signal sequence was ligated in-frame to $\operatorname{tr} x A$, the gene encoding thioredoxin, to create pPetSSTrxA, which expressed a Pet signal sequenceTrxA fusion protein under the control of an IPTG-inducible trc promoter. Western blotting with anti-thioredoxin antibodies of various cellular fractions from E. coli WP570, expressing a DsbA signal sequence-TrxA fusion (DsbASS-TrxA), revealed that the cotranslational SRPdependent DsbA signal sequence could efficiently export thioredoxin to the periplasm, as observed previously (Fig. 3). In contrast, neither the PhoA signal sequence (PhoASS-TrxA) nor the Pet signal sequence (PetSS-TrxA) fusion could direct inner membrane translocation of thioredoxin (Fig. 3). These data strongly suggest that the ESPR-containing signal peptide does not direct cotranslational export via the classical SRP-dependent method, as

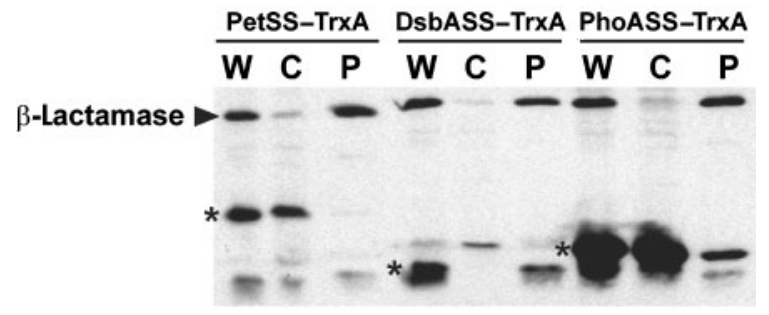

Fig. 3. Export of thioredoxin-signal sequence fusions. Western immunoblot analysis of fractionated E. coli WP570 cells expressing thioredoxin fused to the Pet signal sequence (PetSSTrxA), DsbA signal sequence (DsbASS-TrxA) or PhoA signal sequence (PhoASS-TrxA) is shown. Blots were probed with anti-thioredoxin antibodies and with anti- $\beta$-lactamase antibodies as a control. Like the posttranslationally targeted construct PhoASS-TrxA, the PetSS-TrxA protein was retained in the cytoplasm. In contrast, most of TrxA from the cotranslationally targeted DsbASS-TrxA construct was located in the periplasm. The position of $\beta$-lactamase is indicated, and the positions of the different thioredoxin fusion proteins are indicated by asterisks. Some minor bands are apparent in the strains expressing the PhoASS-TrxA and PetSS-TrxA fusions, the sizes of which correspond well with TrxA lacking a signal sequence. Additionally, there are some minor bands apparent in fractions from the strain possessing the DsbA-TrxA fusion, the sizes of which correspond well with an unprocessed form of the DsbATrxA fusion. W, total cell protein; $\mathrm{P}$, periplasmic fraction; C, cytoplasmic fraction.

previously described (Peterson et al., 2003; Sijbrandi et al., 2003), but that export more closely resembles the posttranslational pathway.

\section{Deletion of the ESPR accelerates translocation of proteins across the inner membrane}

To investigate the contribution of the N1, H1, N2 and $\mathrm{H} 2$ domains to the inner membrane translocation process, deletions were introduced into pQMDSSPet, and the specific activity of PhoA was measured in cellular fractions derived from each construct (Fig. 4). The $\mathrm{H} 2$ domain was shown to be important, as PhoA activity could not be detected from the pQMDSSPet $\Delta \mathrm{I}^{34}-\mathrm{I}^{49}$ construct. In contrast, deletion of the $\mathrm{N} 1$ (pQMDSSPet $\left.\Delta \mathrm{N}^{2}-\mathrm{I}^{7}\right), \mathrm{H} 1$ $\left(\mathrm{pQMDSSPet} \Delta \mathrm{A}^{11}-\mathrm{A}^{23}\right.$ ) or N2 (pQMDSSPet $\Delta \mathrm{K}^{24}-\mathrm{K}^{33}$ ) domains demonstrated that none of these individual domains was absolutely required for PhoA activity, although in the N2 construct, PhoA activity was severely diminished, indicating an important role for this region in inner membrane translocation. Surprisingly, however, protein export was drastically increased when the $\mathrm{N} 1 \mathrm{H} 1$ regions were deleted, either separately or together (pQMDSSPet $\Delta \mathrm{N}^{2}-\mathrm{I}^{7}$, pQMDSSPet $\Delta \mathrm{N}^{2}-\mathrm{A}^{23}$, pQMDSSPet $\Delta \mathrm{N}^{2}-\mathrm{I}^{27}$ and pQMDSSPet $\left.\Delta \mathrm{A}^{11}-\mathrm{A}^{23}\right)$, even though the total cellular levels of PhoA were approximately equivalent, as assessed by Western blotting with anti-PhoA antibodies. Indeed, the efficiency of inner membrane translocation was comparable 
(A)

\begin{tabular}{|c|}
\hline 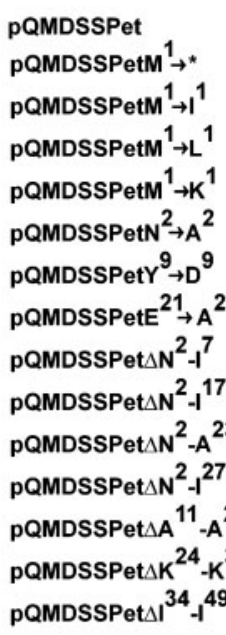 \\
\hline
\end{tabular}

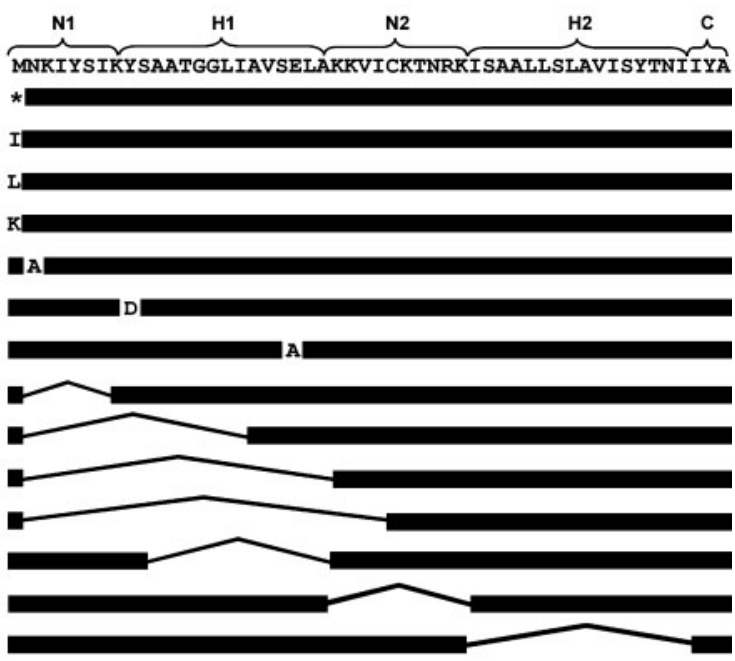

(B)

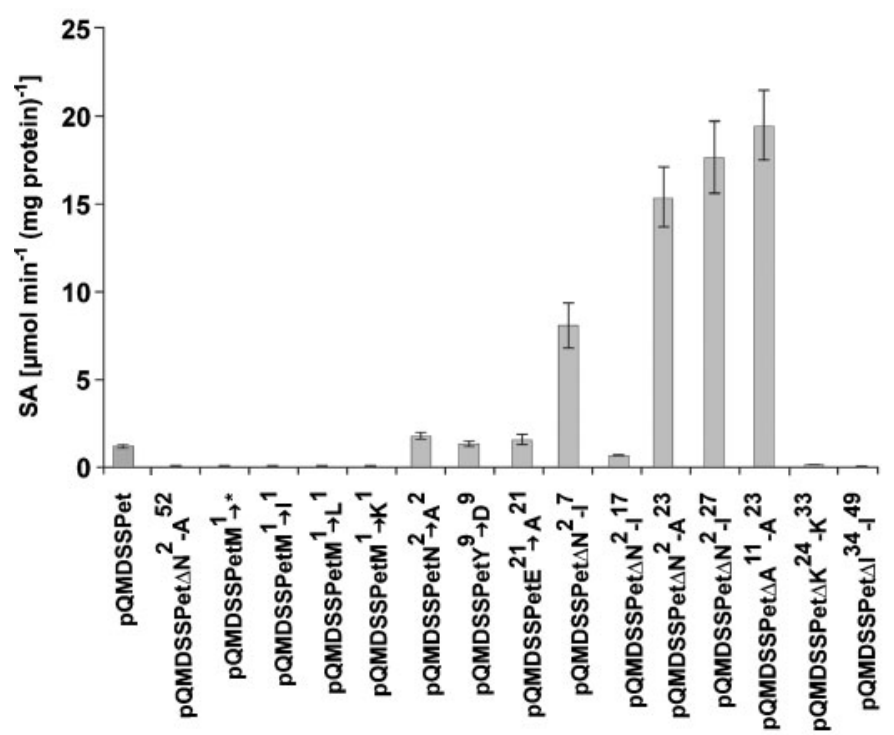

(C)

$\begin{array}{llllllllllllllll}1 & 2 & 3 & 4 & 5 & 6 & 7 & 8 & 9 & 10 & 11 & 12 & 13 & 14 & 15 & 16\end{array}$
Fig. 4. Residues and domains within the Pet signal peptide affect protein export. (A) Various Pet signal peptide mutant constructs made in pQUANTagen $(k x)$; the position of each mutation is shown in relation to the extended signal peptide of Pet. (B) Specific activities (SA) of PhoA expressed from the corresponding signal peptide-PhoA fusion protein constructs indicated in $(A)$. pQMDSSPet was used as a positive control and reference. pQMDSSPet $\Delta \mathrm{N}^{2}-\mathrm{A}^{52}$ was used as a negative control. Data are from three independent experiments, and error bars represent SD. These data demonstrate that constructs lacking the ESPR region were more efficiently translocated to the periplasm than those with a native signal sequence. (C) Western immunoblot analysis of total cellular protein derived from $E$. coli $\Delta$ Star harbouring the various signal peptidePhoA fusion constructs, and probed with anti-PhoA antibodies. Lanes 1-16 correspond to the order of the constructs depicted in (B). These data indicate that the differences in the levels of PhoA activity were independent of the amount of protein present, and were due to the amount of active PhoA translocated to the periplasm. to that obtained with $\mathrm{pQMDSSMBP}{ }^{\star} 1$, an SRP-dependent signal peptide (Fig. 1).

\section{Conserved residues within the ESPR are not essential for directing inner membrane translocation or function}

As mentioned above, the ESPR regions possess remarkable conservation. In order to identify whether the conserved residues are essential for protein translocation across the inner membrane, we performed EP-PCR of the DNA encoding the Pet signal sequence, and fused the signal sequence amplicons to PhoA. Growth of E. coli HB101 containing these constructs on media containing XP allowed selection of mutants permissive (blue) and non-permissive (white) for inner membrane translocation of PhoA. Following sequencing of 192 white colonies arising from 10 independent EP-PCR experiments, no single mutation in the ESPR preventing inner membrane translocation could be detected, with the exception of $\mathrm{M}^{1}$, for which three mutations $\left(\mathrm{M}^{1} \rightarrow \mathrm{I}^{1}, \mathrm{M}^{1} \rightarrow \mathrm{L}^{1}\right.$ and $\left.\mathrm{M}^{1} \rightarrow \mathrm{K}^{1}\right)$ were identified (Fig. 4). When examining the whole Pet signal peptide, the only other amino acid identified that was non-permissive for inner membrane translocation was $\mathrm{L}^{41}$ within the $\mathrm{H} 2$ domain; $\mathrm{L}^{41} \rightarrow \mathrm{P}^{41}$ and $\mathrm{L}^{41} \rightarrow \mathrm{Q}^{41}$ mutations abolished PhoA activity completely. Kyte and Doolittle hydropathy profiling of the signal sequences possessing such mutations demonstrated that these mutations decreased the level of hydrophobicity in the $\mathrm{H} 2$ domain (data not shown). 
To ensure that we had not missed any of the highly conserved residues during our EP-PCR experiments, we used site-directed mutagenesis to make non-conservative mutations within the ESPR region of the Pet signal sequence-PhoA fusion. As expected, mutation of the $\mathrm{N}$ terminal $\mathrm{M}^{1}$ into a stop codon abolished PhoA activity (Fig. 4). Surprisingly, none of the non-conservative mutations, i.e. $\mathrm{N}^{2} \rightarrow \mathrm{A}^{2}, \mathrm{Y}^{9} \rightarrow \mathrm{D}^{9}$ or $\mathrm{E}^{21} \rightarrow \mathrm{A}^{21}$, significantly affected the efficiency of PhoA translocation (Fig. 4).

Since the system we were using is a heterologous system, we could not rule out the possibility that the ESPR conserved residues only play a role in biogenesis of proteins secreted via the T5SS. Thus, we mutated non-conservatively all of the highly conserved residues within the native Pet signal sequence, and monitored by SDS-PAGE analysis of supernatant fractions the ability of these constructs to produce Pet (Fig. 5). Surprisingly, and as with similar mutations within the conserved residues of the signal sequence-PhoA fusions, none of the mutations within the highly conserved residues of the ESPR appeared to significantly affect Pet secretion. However, it should be noted that upon repeated fractionation, the level of Pet released into the culture supernatant was slightly diminished relative to that of the wild-type, when the conserved tyrosine residues were mutated to charged residues $\left(\mathrm{Y}^{5}-\mathrm{R}^{5}\right.$ and $\left.\mathrm{Y}^{9}-\mathrm{R}^{9}\right)$. Previous investigations have suggested that the conserved residues of the ESPR may play a role in the correct folding of the passenger domain. As a marker of correct biogenesis, we tested the functional activity of the protein derived from each site-directed mutant. All mutant forms of Pet demonstrated similar levels of toxicity in the standard HEp-2 cell assay described previously (Eslava et al., 1998; data not shown).

\section{DISCUSSION}

The unusual extended signal sequence was first noted in the TpsA protein FHA, and subsequently among other proteins secreted via the T5SS. The initial hypotheses have suggested that the presence of the ESPR within the unusual signal sequences confers additional functional properties upon the signal sequence, such as: (i) it is required for a specific mode of targeting to the inner membrane, e.g. SRP; (ii) it is required for translocation across the inner membrane via an alternative inner membrane translocon, e.g. Tat; (iii) it is required for the proper folding of the exoproteins; and (iv) it specifically enhances efficient membrane translocation of large exoproteins (Henderson et al., 1998, 2004).

The question of which inner membrane translocation mechanism is utilized by polypeptides possessing an ESPR-containing signal sequence has been the subject of much recent debate. The expression analysis presented in this paper appears to rule out any significant role for the Tat pathway, despite the presence of a motif similar to the Tat motif within some of the signal sequences. In contrast, initial experiments investigating $\mathrm{Hbp}$, a homologue of Pet, indicate that the unusual signal peptide mediates targeting and inner membrane translocation in an SRP-dependent cotranslational manner (Sijbrandi et al., 2003). Further studies using the autotransporter EspP suggest that SRP dependence is due to the inherent hydrophobicity of the signal peptide, and appears not to be associated with the presence of the ESPR per se (Lee \& Bernstein, 2001; Peterson et al., 2003). However, Hbp and EspP contain helix-breaking residues in their H2-domain, which is generally considered to be a discriminatory factor excluding SRP recognition of

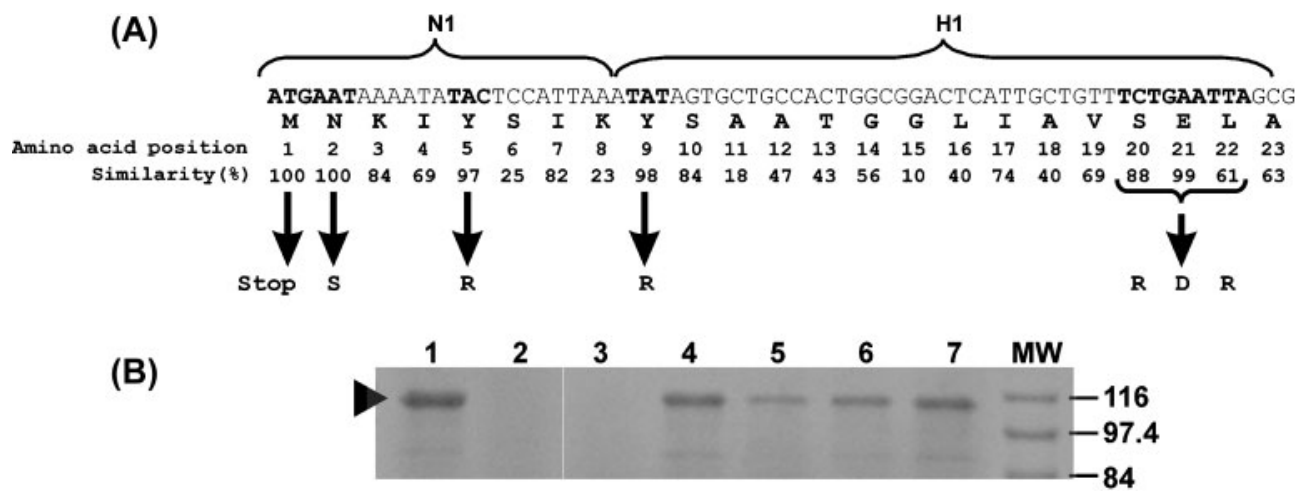

Fig. 5. Site-directed mutagenesis of pet. (A) The nucleotide sequence and corresponding amino acid sequence of the DNA region encoding the ESPR region of the Pet signal peptide. The location of the N1 and H1 domains composing the ESPR are indicated, along with the numbering of each amino acid within the pet ORF. The level of conservation of each amino acid when compared to all known ESPR regions is also shown. The positions of the site-directed mutations are indicated by bold type. The relevant amino acid changes resulting from the mutagenesis are indicated by arrows. (B) SDS-PAGE analysis of culture supernatant fractions harvested after growth of E. coli HB101 containing site-directed mutant derivatives of pCEFN1. Lane 1, wild-type positive control E. coli HB101 pCEFN1; lane 2, negative control E. coli HB101 pSPORT1; lane 3, E. coli

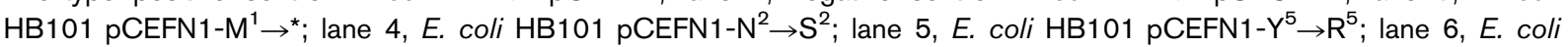
$\mathrm{HB} 101 \mathrm{pCEFN1}-\mathrm{Y}^{9} \rightarrow \mathrm{R}^{9}$; lane 7, E. coli HB101 pCEFN1-S ${ }^{20} \mathrm{EL} \rightarrow \mathrm{R}^{20} \mathrm{DR}$. The sizes of the molecular mass markers (MW) are indicated in $\mathrm{kDa}$. The position of the Pet protein is indicated by an arrowhead. 
signal peptides (Adams et al., 2002). Aware of the bias resulting from the study of SRP dependence using in vivo approaches in which depletion of SRP results in the depletion of SecY, an essential component of the Sec translocon (Beha et al., 2003; Koch et al., 1999; Seluanov \& Bibi, 1997), Chevalier et al. (2004) have investigated the inner membrane translocation of FHA by coupling in vivo and in vitro approaches. Despite transient cross-linking to SRP, it has been concluded that FHA is exported via SecYEG in a posttranslational manner. Indeed, the most recent work from Bernstein's group indicates that the ESPR-containing signal sequence of EspP mediates posttranslational export in a trigger factor-independent fashion (Peterson et al., 2006). The data derived from the TrxA fusion experiments presented here are consistent with a posttranslational route for inner membrane translocation of Pet, and reinforce the recent data generated for FHA and EspP. However, it remains possible that SRP binds the signal sequence and targets the protein to the inner membrane Sec translocon, but that translocation ultimately occurs in a posttranslational fashion. Nevertheless, what remains ultimately clear is that if SRP is involved, translocation does not occur in the classical SRP-dependent cotranslational fashion.

If the ESPR region is not involved in export via the SRP cotranslational pathway, then what is the role for this conserved region in inner membrane translocation? Our results for the Pet signal sequence demonstrated that translocation was less efficient than that associated with signal sequences mediating posttranslational or cotranslational translocation. These data agree well with initial observations for FHA, in which functional investigations have revealed that the unusual signal peptide is a poor mediator of inner membrane translocation (JacobDubuisson et al., 1996). However, data arising from mutational analysis of the Pet signal sequence are striking, revealing that deletion of the ESPR results in a significant increase in the level of PhoA activity. Such a delay in maturation has previously been observed for EspP containing a deletion of the ESPR region (Szabady et al., 2005). However, these authors have speculated that the ESPR might permit temporary inner membrane anchoring, such that the $\beta$-domain of the EspP autotransporter can fold into a correct configuration prior to insertion into the outer membrane (Szabady et al., 2005). Indeed, these investigators have specifically noted that the presence of the ESPR affects neither the rate nor the efficiency of inner membrane translocation (Szabady et al., 2005). However, the theory proposed by Szabady et al. (2005) is difficult to reconcile with the presence of the ESPR in FHA and the other TpsA proteins, where the TpsB outer membrane $\beta$-barrel is secreted separately across the inner membrane, and thus folds independently of the secreted protein (JacobDubuisson et al., 2004). While both studies indicate a delay in maturation, we favour a hypothesis whereby the process of inner membrane translocation is delayed, rather than release of the mature protein from the inner membrane. Previous investigations have demonstrated that PhoA is active within the periplasm, even when tethered to the inner membrane (Manoil \& Beckwith, 1986), and thus it appears that the difference in PhoA activity can only be due to the efficiency of translocation through the SecYEG translocon. In agreement with this finding, recent studies of the FHA ESPR suggest that, in the absence of the ESPR, the secreted protein remains functional, but the presence of the ESPR affects the rate and/or efficiency of inner membrane translocation (Chevalier et al., 2004). Indeed, based on work with EspP, Peterson et al. (2006) now propose that the ESPR mediates an interaction with a cytoplasmic factor, or induces the formation of an unusual signal peptide conformation which delays the onset of protein translocation. Thus, it appears that, rather than having an involvement in a specific mode of inner membrane targeting, the ESPR may simply play a role in regulating the rate of protein export.

The question then arises as to why a specific polypeptide might regulate the rate of inner membrane translocation. In this respect, decreasing the rate of export allows the molecules to fold into a quasi-competent state for subsequent insertion into, and translocation across, the outer membrane. Indeed, the delay observed elsewhere in outer membrane translocation of EspP derivatives lacking the ESPR can alternatively be explained by aggregation of periplasmic intermediates that are translocated across the inner membrane at a high rate in the absence of ESPR (Szabady et al., 2005). However, if the ESPR is absolutely crucial for folding of secreted T5SS proteins, it is difficult to explain why it is not systematically present in all such proteins. On the other hand, overexpression of autotransporters is often detrimental to bacterial cells (Henderson \& Desvaux, 2004), and the presence of the ESPR may prevent rapid accumulation of periplasmic intermediates to a toxic level.

Nevertheless, these data raise several possibilities for the role of the ESPR in inner membrane translocation. One such possibility is that the ESPR-containing signal sequences target proteins posttranslationally, but delay interaction of the signal peptide with the SecYEG translocon by adopting a specific conformation. The importance of secondary structure within signal sequences is well established and again demonstrated here, where a mutation disrupting the secondary structure, such as mutation of $\mathrm{L}^{41}$ or deletion of any domain, greatly influences translocation.

Alternatively, the ESPR may delay translocation of the polypeptide through the SecYEG translocon. This observation is supported by the delay in accumulation of active PhoA in the periplasm, and by other data which demonstrate that overproduction of a protein containing an ESPR-type signal sequence delays export of other proteins when expressed in trans (Szabady et al., 2005). However, if the mechanism behind decreased protein export depends only on the presence of a transmembrane helix within ESPR, it is difficult to explain why this domain is so 
conserved, and why mutation of the conserved residues does not appear to affect inner membrane translocation.

A third possibility is that the presence of the ESPR permits recognition by a specific cytoplasmic or inner-membrane protein(s) which slows down protein translocation. While debate still continues over SRP involvement, there remains the possibility that other cytoplasmic or inner-membrane factors are also involved. In fact, global mutagenesis strategies employed by us and others have identified several cytoplasmic and inner membrane proteins which appear to play a role in biogenesis of proteins secreted via the T5SS (Hardie et al., 2004; M. Desvaux and others, unpublished results).

In conclusion, a consensus opinion is now emerging that inner membrane translocation of proteins possessing an ESPR-type signal peptide occurs in a posttranslational fashion, and the data presented here argue against the proposition that these proteins must be translocated through the SecYEG translocon in a cotranslational fashion to avoid degradation or folding within the cytoplasm. Furthermore, these data indicate that the ESPR delays inner membrane translocation by hindering interaction with the SecYEG translocon, or by delaying passage through the translocon in a novel fashion.

\section{ACKNOWLEDGEMENTS}

Work in the Henderson laboratory was supported by Biotechnology and Biological Sciences Research Council (BBSRC) grants 81/D14955 and $81 /$ P14130 to I. R. H. Work in the Nataro laboratory was funded under US Public Health Service grant AI43617 to J.P.N.D.H. is supported by US Public Health Service grant GM41883 to Jon Beckwith. We thank Chris Bailey for his help in constructing the figures, Harris Bernstein for help with the SecA depletion experiments, and Jon Beckwith for providing strains. DNA sequencing was performed at the Functional Genomics and Proteomics Laboratory of the University of Birmingham.

\section{REFERENCES}

Adams, H., Scotti, P. A., De Cock, H., Luirink, J. \& Tommassen, J. (2002). The presence of a helix breaker in the hydrophobic core of signal sequences of secretory proteins prevents recognition by the signal-recognition particle in Escherichia coli. Eur J Biochem 269, 5564-5571.

Beha, D., Deitermann, S., Muller, M. \& Koch, H. G. (2003). Export of beta-lactamase is independent of the signal recognition particle. J Biol Chem 278, 22161-22167.

Bogsch, E. G., Sargent, F., Stanley, N. R., Berks, B. C., Robinson, C. \& Palmer, T. (1998). An essential component of a novel bacterial protein export system with homologues in plastids and mitochondria. J Biol Chem 273, 18003-18006.

Bradford, M. M. (1976). A rapid and sensitive method for the quantitation of microgram quantities of protein utilizing the principle of protein-dye binding. Anal Biochem 72, 248-254.

Chevalier, N., Moser, M., Koch, H. G., Schimz, K. L., Willery, E., Locht, C., Jacob-Dubuisson, F. \& Muller, M. (2004). Membrane targeting of a bacterial virulence factor harbouring an extended signal peptide. J Mol Microbiol Biotechnol 8, 7-18.

Cotter, S. E., Surana, N. K. \& St Geme, J. W., 3rd (2005). Trimeric autotransporters: a distinct subfamily of autotransporter proteins. Trends Microbiol 13, 199-205.

Cristobal, S., de Gier, J. W., Nielsen, H. \& von Heijne, G. (1999). Competition between Sec- and TAT-dependent protein translocation in Escherichia coli. EMBO J 18, 2982-2990.

DeLisa, M. P., Tullman, D. \& Georgiou, G. (2003). Folding quality control in the export of proteins by the bacterial twin-arginine translocation pathway. Proc Natl Acad Sci U S A 100, 6115-6120.

Desvaux, M., Parham, N. J. \& Henderson, I. R. (2004a). Type V protein secretion: simplicity gone awry? Curr Issues Mol Biol 6, 111-124.

Desvaux, M., Parham, N. J. \& Henderson, I. R. (2004b). The autotransporter secretion system. Res Microbiol 155, 53-60.

Desvaux, M., Cooper, L. C., Filenko, N. A., Scott-Tucker, A., Turner, S. M., Cole, J. A. \& Henderson, I. R. (2006). The unusual signal sequence of the Type $\mathrm{V}$ secretion is phylogenetically restricted. FEMS Microbiol Lett 264, 22-30.

Eslava, C., Navarro-Garcia, F., Czeczulin, J. R., Henderson, I. R., Cravioto, A. \& Nataro, J. P. (1998). Pet, an autotransporter enterotoxin from enteroaggregative Escherichia coli. Infect Immun 66, 3155-3163.

French, C., Keshavarz-Moore, E. \& Ward, J. M. (1996). Development of a simple method for the recovery of recombinant proteins from the Escherichia coli periplasm. Enzyme Microb Technol 19, 332-338.

Hardie, K. R., Arnold, L., Dodson, S. \& Baldwin, T. (2004). Autotransporters do not reach their extracellular location alone. In Abstracts of the 155th Meeting of the Society for General Microbiology, 6-9 September 2004, Trinity College Dublin, abstract no. CCS 28. Reading, UK: Society for General Microbiology. http://www.sgm. ac.uk/meetings/pdfabstracts/tcd2004abs.pdf

Henderson, I. R. \& Desvaux, M. (2004). The type V secretion pathway: a premium source of virulence factors? Drug Discov Today 9, 240-243.

Henderson, I. R. \& Nataro, J. P. (2001). Virulence functions of autotransporter proteins. Infect Immun 69, 1231-1243.

Henderson, I. R., Navarro-Garcia, F. \& Nataro, J. P. (1998). The great escape: structure and function of the autotransporter proteins. Trends Microbiol 6, 370-378.

Henderson, I. R., Navarro-Garcia, F., Desvaux, M., Fernandez, R. C. \& Ala'Aldeen, D. (2004). Type V protein secretion pathway: the autotransporter story. Microbiol Mol Biol Rev 68, 692-744.

Huber, D., Boyd, D., Xia, Y., Olma, M. H., Gerstein, M. \& Beckwith, J. (2005). Use of thioredoxin as a reporter to identify a subset of Escherichia coli signal sequences that promote signal recognition particle-dependent translocation. J Bacteriol 187, 2983-2991.

Jacob-Dubuisson, F., Locht, C. \& Antoine, R. (2001). Two-partner secretion in Gram-negative bacteria: a thrifty, specific pathway for large virulence proteins. Mol Microbiol 40, 306-313.

Jacob-Dubuisson, F., Buisine, C., Mielcarek, N., Clement, E., Menozzi, F. D. \& Locht, C. (1996). Amino-terminal maturation of the Bordetella pertussis filamentous haemagglutinin. Mol Microbiol 19, 65-78.

Jacob-Dubuisson, F., Fernandez, R. \& Coutte, L. (2004). Protein secretion through autotransporter and two-partner pathways. Biochim Biophys Acta 1694, 235-257.

Koch, H. G., Hengelage, T., Neumann-Haefelin, C., MacFarlane, J., Hoffschulte, H. K., Schimz, K. L., Mechler, B. \& Muller, M. (1999). In vitro studies with purified components reveal signal recognition 
particle (SRP) and SecA/SecB as constituents of two independent protein-targeting pathways of Escherichia coli. Mol Biol Cell 10, 2163-2173.

Laemmli, U. K. (1970). Cleavage of structural proteins during the assembly of the head of bacteriophage T4. Nature 227, 680-685.

Lee, H. C. \& Bernstein, H. D. (2001). The targeting pathway of Escherichia coli presecretory and integral membrane proteins is specified by the hydrophobicity of the targeting signal. Proc Natl Acad Sci U S A 98, 3471-3476.

Manoil, C. \& Beckwith, J. (1986). A genetic approach to analyzing membrane protein topology. Science 233, 1403-1408.

Meng, G., Surana, N. K., St Geme, J. W., III \& Waksman, G. (2006). Structure of the outer membrane translocator domain of the Haemophilus influenzae Hia trimeric autotransporter. EMBO J 25, 2297-2304.

Mougous, J. D., Cuff, M. E., Raunser, S., Shen, A., Zhou, M., Gifford, C. A., Goodman, A. L., Joachimiak, G., Ordonez, C. L. \& other authors. (2006). A virulence locus of Pseudomonas aeruginosa encodes a protein secretion apparatus. Science 312, $1526-1530$.

Newman, C. L. \& Stathopoulos, C. (2004). Autotransporter and two-partner secretion: delivery of large-size virulence factors by gram-negative bacterial pathogens. Crit Rev Microbiol 30, 275-286.

Peterson, J. H., Woolhead, C. A. \& Bernstein, H. D. (2003). Basic amino acids in a distinct subset of signal peptides promote interaction with the signal recognition particle. J Biol Chem 278, 46155-46162.

Peterson, J. H., Szabady, R. L. \& Bernstein, H. D. (2006). An unusual signal peptide extension inhibits the binding of bacterial presecretory proteins to the signal recognition particle, trigger factor and the SecYEG complex. J Biol Chem 281, 9038-9048.

Richter, S. \& Bruser, T. (2005). Targeting of unfolded PhoA to the Tat translocon of Escherichia coli. J Biol Chem 280, 42723-42730.
Sambrook, J. \& Russell, D. W. (2001). Molecular Cloning: a Laboratory Manual, 3rd edn. Cold Spring Harbor, NY: Cold Spring Harbor Laboratory.

Schierle, C. F., Berkmen, M., Huber, D., Kumamoto, C., Boyd, D. \& Beckwith, J. (2003). The DsbA signal sequence directs efficient, cotranslational export of passenger proteins to the Escherichia coli periplasm via the signal recognition particle pathway. J Bacteriol 185, 5706-5713.

Seluanov, A. \& Bibi, E. (1997). FtsY, the prokaryotic signal recognition particle receptor homologue, is essential for biogenesis of membrane proteins. J Biol Chem 272, 2053-2055.

Sijbrandi, R., Urbanus, M. L., ten Hagen-Jongman, C. M., Bernstein, H. D., Oudega, B., Otto, B. R. \& Luirink, J. (2003). Signal recognition particle (SRP)-mediated targeting and Sec-dependent translocation of an extracellular Escherichia coli protein. J Biol Chem 278, 4654-4659.

Surana, N. K., Cutter, D., Barenkamp, S. J. \& St Geme, J. W., III (2004). The Haemophilus influenzae Hia autotransporter contains an unusually short trimeric translocator domain. J Biol Chem 279, 14679-14685.

Szabady, R. L., Peterson, J. H., Skillman, K. M. \& Bernstein, H. D. (2005). An unusual signal peptide facilitates late steps in the biogenesis of a bacterial autotransporter. Proc Natl Acad Sci U S A 102, 221-226.

Thanassi, D. G., Stathopoulos, C., Karkal, A. \& Li, H. (2005). Protein secretion in the absence of ATP: the autotransporter, two-partner secretion and chaperone/usher pathways of Gram-negative bacteria (review). Mol Membr Biol 22, 63-72.

Ulbrandt, N. D., Newitt, J. A. \& Bernstein, H. D. (1997). The E. coli signal recognition particle is required for the insertion of a subset of inner membrane proteins. Cell 88, 187-196.

Walter, K. \& Schutt, C. (1976). Methods of Enzymatic Analysis, 2nd edn. New York: Academic Press.

Edited by: S. MacIntyre 